\title{
DESTRUCTIBLE AND INDESTRUCTIBLE BLASCHKE PRODUCTS
}

BY

\author{
H. STEPHEN MORSE
}

\begin{abstract}
A special case of destructibility for Blaschke products is introduced and studied. An example is given of a destructible Blaschke product which becomes indestructible when a single point is deleted from its zero-set.
\end{abstract}

1. Introduction. Let $\Delta$ denote the open unit disk, $\{|z|<1\}$, and let $C=$ fr $\Delta$ $=\{|z|=1\}$. If $f$ is analytic on $\Delta$, we say that $f$ is an inner function provided that $|f(z)|<1, z \in \Delta$, and that $f^{*}(\zeta) \in C$ for almost all $\zeta \in C$. Here, $f^{*}$ denotes the extension of $f$ via the Fatou sectorial limit. It is known that every Blaschke product is inner, and that if $f$ is inner, we may write $f=B g$, where $B$ is the Blaschke product arising from the zeros of $f$ and where $g$ is a nonvanishing inner function.

For $a \in \Delta$, let $L_{a}(z)=(a-z) /(1-\bar{a} z), z \in \Delta$. We recall that $L_{a}$ is a conformal automorphism of $\Delta$, and is its own inverse. Since $L_{a}$ preserves $C$, we see that $L_{a} \circ f$ is inner whenever $f$ is. In his thesis [2], Frostman showed that $L_{a} \circ f$ is a Blaschke product for all points, $a$, in $\Delta$ lying off an exceptional set of capacity zero (perhaps empty). For certain inner functions, it may happen that the exceptional set in Frostman's theorem is empty. That is, we may have that $L_{a} \circ f$ is a Blaschke product for every point, $a$, in $\Delta$. Since $L_{0} \circ f=-f$, we see that $f$ is itself a Blaschke product, and in this case we say that $f$ is an indestructible Blaschke product. Clearly, every finite Blaschke product is indestructible. If $B$ is a Blaschke product whose exceptional set is not empty, we say that $B$ is a descructible Blaschke product. This terminology is due to McLaughlin [4], although the notions in question were introduced and studied earlier by Heins [3].

The problem taken up in the present paper is that of characterizing the zero-set of an indestructible Blaschke product. In [4], McLaughlin has given a necessary and sufficient condition for a Blaschke product, $B$, to be indestructible. However, the criterion given is in terms of the $a$-points of $B$ for each point, $a$, in $\Delta$, and is thus at the very least cumbersome in specific applications. Since a Blaschke product is completely specified (up to a harmless rotation) by its zero-set, a more natural and useful characterization would be one solely in terms of the zero-set. Thus, a possible "good" solution might be

Received by the editors October 27, 1978.

AMS (MOS) subject classifications (1970). Primary 30A76.

Key words and phrases. Blaschke product, inner function, singular harmonic function, minimal harmonic function, Poisson-Stielties integral representation. 
a geometrical condition on the zeros of $B$ which would guarantee indestructibility, and conversely. While we are not able to present a solution for this problem, the present paper culminates in an example of a destructible Blaschke product which becomes indestructible when a single point is deleted from its zero-set (see Proposition 4.1). The example thus rules out several lines of attack, and suggests to that extent that a full solution may be fairly difficult.

The methods we use are potential-theoretic. In particular, the PoissonStieltjes integral representation for nonnegative harmonic functions on $\Delta$ is a major tool. In $\S 3$, we isolate a particular instance of destructibility for special study, and show that this leads to an exponential growth condition which is lost whenever one of the zeros is deleted. This preliminary investigation is complemented by a deep result of de la Vallée-Poussin on continuous singular monotone functions, at which point, in $\$ 4$, the example cited above easily follows. We conclude, in $\$ 5$, with some heuristic comments concerning the "thinness" of the set of destructible Blaschke products.

This paper represents one section of my dissertation (University of Maryland, College Park, May 1978). My thanks are due to Professor Maurice Heins for his guidance and advice in the research presented here.

2. Singular harmonic functions and inner functions. We denote by $\mathscr{P}$ the collection of all nonnegative harmonic functions on $\Delta$. For each $u \in \mathscr{P}$, there exists a monotone nondecreasing function, $\mu$, such that

$$
u(z)=\frac{1}{2 \pi} \int_{0}^{2 \pi} k(\theta, z) d \mu(\theta), \quad z \in \Delta,
$$

where $k(\theta, z)=\operatorname{Re}\left\{\left(e^{i \theta}+z\right) /\left(e^{i \theta}-z\right)\right\}$ is the Poisson kernel. Formula (2.1) is called the Poisson-Stieltjes integral representation for $u$. The existence of such a representation is due to Riesz [7]. The celebrated Fatou theorem [1] guarantees the equality of $u^{*}\left(e^{i \theta}\right)$ and $\mu^{\prime}(\theta)$ whenever $\mu^{\prime}(\theta)$ exists (finite or infinite). Thus, if $\mu$ is singular (i.e., if $\mu^{\prime}=0$ a.e.), then $u^{*}$ vanishes almost everywhere on $C$. In this case, $u$ is said to be a singular member of $\mathscr{P}$ (in the sense of Parreau [6]). Such a function can be characterized by the property that it dominates no nonvanishing bounded member of $\mathscr{P}$.

For $\eta=e^{i \theta} \in C$, we denote by $K_{\eta}$ the member of $\mathcal{P}$ given by $K_{\eta}(z)=$ $k(\theta, z)$. We note that $K_{\eta}$ is a singular member of $\mathcal{P}$ for each $\eta \in C$ (in fact, $K_{\eta}$ vanishes continuously on $\left.C \backslash\{\eta\}\right)$. The positive multiples of $K_{\eta}$ are the minimal harmonic functions on $\Delta$ (in the sense of R. S. Martin [5]), since they have the property that any harmonic minorant of $K_{\eta}$ is necessarily proportional to it. They arise from the discontinuities of $\mu$ (the generating function) in that a discontinuity of $\mu$ at $\theta_{0}$ results (up to a positive multiple) in an evaluation of $\theta \rightarrow k(\theta, z)$ at $\theta_{0}$. Suppose now that $u \in \mathcal{P}$ is singular with generating function $\mu$. Thus, $\mu$ is a singular monotone function, and we may separate $\mu$ into its continuous and discrete parts. That is, we may write $u=T+s$, where $T$ is a sum of minimals arising from the (at most countable) discontinuities of $\mu$, and where $s$ has a continuous singular monotone 
generating function. We shall say that a member of $\mathscr{P}$ whose generating function is continuous and singular is $C$-singular, although this terminology is not standard.

A deep result due to de la Vallée-Poussin ([9, pp. 88-92]; also see [8, p. 128]) guarantees the derivative of a nonconstant continuous singular monotone function on an interval is $+\infty$ at an uncountable set of points of the interval. Invoking the Fatou theorem, we obtain

LEMMA 2.1. Let $u$ be a singular member of $\mathscr{P}$. Then $u$ is a pure sum of minimals provided that $u^{*}(\zeta)=+\infty$ at at most a countable set of points, $\zeta$, of C.

Proof of Lemma 2.1. We write $u=T+s$, where $T$ is a sum of minimals and $s$ is $C$-singular. If $\sigma$ is the generating function of $s$, and $\sigma$ is not constant, then $\sigma^{\prime}(\theta)=+\infty$ for uncountably many $\theta \in[0,2 \pi]$. The Fatou theorem then implies that $u^{*}\left(e^{i \theta}\right)>s^{*}\left(e^{i \theta}\right)=\sigma^{\prime}(\theta)=+\infty$ at these points, contradicting the assumption on $u$. Hence, $\sigma$ is constant, and $s=0$, to complete the proof.

Singular harmonic functions arise naturally from inner functions in the following way. If $f$ is inner, we recall that we may write $f=B g$, where $B$ is a Blaschke product arising from the zeros of $f$, and where $g$ is a nonvanishing inner function. We consider the nonnegative superharmonic function $-\log |f|$, and its greatest harmonic minorant (denoted by “ $m$ "). We have

$$
m(-\log |f|)=m(-\log |B|)+m(-\log |g|)=-\log |g|,
$$

since " $m$ " is additive, $m(-\log |B|)=0$, and $g$ is nonvanishing. Since $g$ is inner, we see that $(-\log |g|)^{*}(\zeta)=-\log \left|g^{*}(\zeta)\right|=0$ for almost all $\zeta$ in $C$, and hence that $m(-\log |f|)$ is singular (perhaps zero). Since $f$ will be a pure Blaschke product if and only if $g$ is a unimodular constant, we obtain

LEMMA 2.2. Let $f$ be an inner function. Then the function $m(-\log |f|)$ is singular, and $f$ is a Blaschke product if and only if $m(-\log |f|)=0$.

We remark that no claims of originality are made for the lemmas stated above. In particular, Lemma 2.2 is a special case of much more general results due to Heins [3]. Although Lemma 2.1 seems not to appear in this form in the literature, it is little more than an observation, and should be familiar to the specialist.

3. Exponential destructibility. For $f$ an inner function and $a \in \Delta$, we define a member of $\mathcal{P}$, denoted by $u_{a}$, by the relation $u_{a}=m\left(-\log \left|L_{a} \circ f\right|\right)$. By Lemma 2.2, $L_{a} \circ f$ is a Blaschke product if and only if $u_{a}=0$, and we see that the exceptional set in Frostman's theorem is given by $\left\{a \in \Delta \mid u_{a}>0\right\}$. Since $u_{a}$ is singular, we may write $u_{a}=T_{a}+s_{a}$, where $T_{a}$ is a sum of minimals and $s_{a}$ is $C$-singular. Clearly, $T_{a}>0$ forces $u_{a}>0$, so that $a$ is an exceptional point. This leads to the following definition. For $B$ a Blaschke product and $\eta \in C$, we say that $B$ is exponentially destructible at $\eta$ provided that $u_{a}$ 
dominates a minimal with singularity at $\eta$. That is, there must exist $a \in \Delta$ and $\beta>0$ such that $u_{a}>\beta K_{\eta}$.

Suppose that $B$ is a Blaschke product, and that $B$ is exponentially destructible at $\eta \in C$. Then $\beta K_{\eta}<m\left(-\log \left|L_{a} \circ B\right|\right)$ for some $a \in \Delta$, and we see that $B^{*}(\eta)=a$, from which it follows that the point $a$ is unique. Further, if we set $\beta=\sup \left\{\alpha \mid \alpha K_{\eta}<u_{a}\right\}$, we see that "sup" may be replaced by "max,", and that $\beta$ so chosen is unique. In fact, $\beta$ is characterized by the property that $\left(u_{a}-\beta K_{\eta}\right) \in \mathcal{P}$ and does not dominate any minimal with singularity at $\eta$. Let $Q(B)$ denote the set of all $\eta \in C$ for which $B$ is exponentially destructible at $\eta$.

Proposition 3.1. For each $\eta \in Q(B)$, there exists a unique ordered pair $[a(\eta), \beta(\eta)] \in \Delta \times\left(\mathbf{R}^{+} \backslash\{0\}\right)$ such that $\left(u_{a(\eta)}-\beta(\eta) K_{\eta}\right) \in \mathscr{P}$ and dominates no minimal with singularity at $\eta$.

Proposition 3.1 is immediate from the remarks which precede it. Note that if $\eta \in Q(B),\left|B^{*}(\eta)\right|=|a(\eta)|<1$. Since $\left|B^{*}(\eta)\right|=1$ for almost all $\eta \in C$, we see that the (linear Lebesgue) measure of $Q(B)$ is zero. Also, if $u_{a}$ dominates more than one minimal, we can have $\eta_{1}$ and $\eta_{2}$ in $Q(B)$ with $\eta_{1} \neq \eta_{2}$, but $a\left(\eta_{1}\right)=a\left(\eta_{2}\right)$.

The following proposition gives a useful representation for exponentially destructible Blaschke products.

Proposition 3.2. Let $B$ be a Blaschke product, and let $\eta \in Q(B)$. Let $(a, \beta)=[a(\eta), \beta(\eta)]$. Then there exists an inner function, $g$, such that

$$
L_{a} \circ B(z)=g(z) \exp (-\beta(\eta+z) /(\eta-z)) .
$$

Proof of Proposition 3.2. Let $v=u_{a}-\beta K_{\eta}$; we see that $v \in \mathscr{P}$. Let $\tilde{v}$ be the harmonic conjugate of $v$ on $\Delta$ satisfying $\tilde{v}(0)=0$, and let $B_{a}$ be the Blaschke product formed from the $a$-points of $B$ (i.e., the zeros of $L_{a} \circ B$ ). Consider the function $\varphi$ defined by

$$
\varphi(z)=B_{a}(z) \exp \{-[v(z)+i \tilde{v}(z)]\} \exp \left(-\beta \frac{\eta+z}{\eta-z}\right) .
$$

We have

$$
-\log |\varphi|=-\log \left|B_{a}\right|+v+\beta K_{\eta}=-\log \left|B_{a}\right|+u_{a}=-\log \left|L_{a} \circ B\right| .
$$

From (3.3), we see upon exponentiating that

$$
\begin{aligned}
L_{a} \circ B(z) & =\alpha B_{a}(z) \exp \{-[v(z)+i \tilde{v}(z)]\} \exp \left(-\beta \frac{\eta+z}{\eta-z}\right) \\
& =g(z) \exp \left(-\beta \frac{\eta+z}{\eta-z}\right),
\end{aligned}
$$

where $|\alpha|=1$ and $g=\alpha B_{a} \exp [-(v+i \tilde{v})]$. Now $\alpha B_{a}$ is inner, since $B_{a}$ is a Blaschke product. Further, since $v$ is singular, $v^{*}$ vanishes almost everywhere on $C$, and we see that $\exp [-(v+i \tilde{v})]$ is inner. Thus, $g$ is inner, and the proof is complete. 
It is the presence of the exponential factor in (3.1) which motivates the terminology "exponential destructibility". We also note, for the sake of completeness, that Proposition 3.2 has a converse. Namely, suppose that a Blaschke product, $B$, satisfies (3.1) for some inner function, $g$, and some point $a \in \Delta$ and some $\beta>0$. Then $\eta \in Q(B)$, as follows immediately from

$$
-\log \left|L_{a} \circ B\right|=-\log |g|+\beta K_{\eta}>\beta K_{\eta} .
$$

LEMMA 3.3. Let $B$ be a Blaschke product, $\eta \in C$, and $a \in \Delta$. Let $D(r)=$ $|B[(1-r) \eta]-a|, 0<r<1$. If $\eta \in Q(B)$ and $a=a(\eta)$, then $D(r)=$ $\mathcal{O}\left(e^{-\alpha / r}\right)$ as $r \rightarrow 0$ for $0<\alpha<2 \beta(\eta)$.

Proof of Lemma 3.3. Using the representation for $B$ provided by (3.1), we find that $D(r)=e^{-2 \beta / r} P(r)$, where $P(r)$ is bounded as $r \rightarrow 0$. Lemma 3.3 now follows.

We are now in a position to consider the effect of adjoining points to the zero-set of a Blaschke product.

Proposition 3.4. Let $B$ be a Blaschke product and $c \in \Delta$. Then $Q(B) \cap$ $Q\left(L_{c} B\right)=\varnothing$.

Proof of Proposition 3.4. We suppose that $\eta \in Q(B)$, and show that $\eta \notin Q\left(L_{c} B\right)$. Let $a=a(\eta)$. Since $Q\left(\zeta L_{c} B\right)=Q\left(L_{c} B\right)$ for any $\zeta \in C$, it suffices to show that $\eta \notin Q(\tilde{B})$, where $\tilde{B}=\left[L_{c}(\eta)\right]^{-1} L_{c} B$. Note that $(\tilde{B})^{*}(\eta)=$ $B^{*}(\eta)=a$.

Suppose, contrary to the assertion, that $\eta \in Q(\tilde{B})$. Let $\tilde{D}(r)=\mid \tilde{B}[(1-r) \eta]$ - $a$. By Lemma 3.3, we would have $\tilde{D}(r)=\theta\left(e^{-\alpha / r}\right)$ as $r \rightarrow 0$ for some $\alpha>0$. However, using the representation (3.1) for $B$ and the definition of $\tilde{B}$, we find that $\tilde{D}(r) \neq \theta\left(e^{-\alpha / r}\right)$ as $r \rightarrow 0$ for any $\alpha>0$. We conclude that $\eta \notin Q(\tilde{B})$, and the proof is complete.

The following corollary follows immediately.

Corollary to Proposition 3.4. Let $B$ be a Blaschke product, and let $c$ be $a$ zero of $B$. Then $Q(B) \cap Q\left(B / L_{c}\right)=\varnothing$.

The corollary is to be interpreted as saying that exponential destructibility at a point of $C$ is lost when any of the zeros of the Blaschke product is deleted. It is in this form we shall use Proposition 3.4, although other versions are readily stated. The following questions merit further study. We have seen that a necessary condition for exponential destructibility is that $D(r)=$ $\theta\left(e^{-\alpha / r}\right)$ for some $\alpha>0$. The question is, is this condition also sufficient. An affirmative answer would be particularly helpful with the following problem: construct a Blaschke product, $B$, such that for some point $c \in \Delta$, both $Q(B)$ and $Q\left(L_{c} B\right)$ are nonempty.

4. An example. We are now prepared to construct an example of a Blaschke product which is destructible, but which becomes indestructible when a single point is deleted from its zero-set. We reason as follows. For a Blaschke 
product, $B$, and $a \in \Delta$, we consider $u_{a}=m\left(-\log \left|L_{a} \circ B\right|\right)$, and write $u_{a}=T_{a}$ $+s_{a}$, where $T_{a}$ is a sum of minimals and $s_{a}$ is $C$-singular. Suppose that $\left|B^{*}(\eta)\right|<1$ for at most countably many points, $\eta$, of $C$. Then $u_{a}^{*}(\eta)=+\infty$ for at most countably many $\eta \in C$, and we see by Lemma 2.1 that $s_{a}=0$ for all points $a \in \Delta$. In particular, $u_{a}=T_{a}$, and we see that if $u_{a}>0$, then $a=a(\eta)$ for some $\eta \in Q(B)$ (i.e., $Q(B) \neq \varnothing$ ). Briefly the countability of $\left\{\eta \in C|| B^{*}(\eta) \mid<1\right\}$ forces $s_{a}$ to be identically zero, in which case "destructible" implies "exponentially destructible".

Now Blaschke products, $B$, for which $\left\{\eta \in C|| B^{*}(\eta) \mid<1\right\}$ is countable are easily constructed. In particular, let $F$ be the set of accumulation points of zeros of $B$. Then $B$ has a continuous extension to $C \backslash F$ on which it takes values of modulus 1 . That is

$$
Q(B) \subset\left\{\eta \in C|| B^{*}(\eta) \mid<1\right\} \subset F .
$$

The countability of $F$ thus places us in the required position.

An interesting special case occurs when $F$ consists of a single point, $\eta$. If $u_{a}>0$ for some point $a \in \Delta$, we have that $B^{*}(\eta)=a$, and hence $\eta \in Q(B)$. Further, for any $\zeta \in C \backslash\{\eta\}, B^{*}(\zeta) \in C$, so that $\zeta \notin Q(B)$. We conclude that if $B$ is destructible, the exceptional set for $B$ consists of a single point, and that $Q(B)=\{\eta\}$.

Proposition 4.1. There exists a destructible Blaschke product which becomes indestructible when one point is deleted from its zero set.

Proof of Proposition 4.1. Choose $a \in \Delta \backslash\{0\}$, and define $B$ by

$$
B(z)=L_{a}[\exp (-(1+z) /(1-z))], \quad z \in \Delta .
$$

We see that $B: \Delta \rightarrow \Delta$; further, $B^{*}$ is continuous and of modulus 1 on $C \backslash\{1\}$, and $\left|B^{*}(1)\right|=|a| \neq 0$. Hence, $m(-\log |B|)=0$, and $B$ is a Blaschke product by Lemma 2.2. Further, $1 \in Q(B)$, as we see by taking $L_{a}$ of both sides in (4.2) and recalling that $L_{a}$ is its own inverse. Let $c$ be any zero of $B$, and define $\tilde{B}(z)=B(z) / L_{c}(z)$. By the corollary to Proposition 3.4, we have that $\{1\} \notin Q(\tilde{B})$. However, $Q(\tilde{B})$ is contained in the cluster set of the zeros of $\tilde{B}$, which is $\{1\}$. Thus, $Q(\tilde{B})=\varnothing$, and $B$ is not exponentially destructible at any point of $C$. By the remarks made at the beginning of this section, if $\tilde{B}$ were destructible, $Q(\tilde{B})$ could not be empty. We see that $\tilde{B}$ is indestructible, and the proof is complete.

It is worth noting that the same reasoning can be used whenever $F$ is at most countable and $F=Q(B)$. For, as above, we have $Q(\tilde{B}) \subset F$ and $Q(B) \cap Q(\tilde{B})=\varnothing$, from which it follows that $Q(\tilde{B})=\varnothing$. Also, the argument holds when a zero is adjoined to the zero-set of $B$, since this amounts to multiplication by $L_{c}$ for some point $c \in \Delta$, and this case is covered by Proposition 3.4.

5. Conclusion. Proposition 4.1 is important insofar as it indicates that a purely geometrical resolution of the problem of characterizing the zero-set of an indestructible Blaschke product will be, persumably, hard to come by. 
Indeed, such a criterion must necessarily be sensitive to the presence or absence of single points in the set of zeros of $B$.

We conclude with the following heuristic comment. By an argument exactly analogous to that given in Proposition 3.4, we can show that exponential destructibility is lost by "moving" one of the zeros of $B$. Such a modification is accomplished by dividing $B$ by $L_{c}$, where $c$ is a zero of $B$, and then multiplying by some $L_{d}$ where $d \neq c$. This suggests that an arrangement of zeros for a Blaschke product which gives rise to exponential destructibility is quite rigid. Or, heuristically, that Blaschke products for which at least exponential destructibility occurs constitute a "thin" set, in some appropriate sense. Clearly, much remains to be done in clarifying these matters and making them more precise.

\section{REFERENCES}

1. P. Fatou, Séries trigonometriques et séries de Taylor, Acta Math. 30 (1906), 335-400.

2. O. Frostman, Potential d'équilibre et capacité des ensembles avec quelques applications à la théorie des fonctions, Medd. Lunds Univ. Mat. Sem. 3 (1935), 1-115.

3. M. Heins, On the Lindelö principle, Ann. of Math. 61 (1953), 440-473.

4. R. McLaughlin, Exceptional sets for inner functions, J. London Math. Soc. 4 (1972), 696-700.

5. R. S. Martin, Minimal positive harmonic functions, Trans. Amer. Math. Soc. 49 (1941), 137-172.

6. M. Parreau, Sur les moyennes des fonctions harmoniques et la classification des surfaces de Riemann, Ann. Inst. Fourier (Grenoble) 3 (1951), 103-197.

7. F. Riesz, Sur certains systèmes singuliers d'équations intégrales, Ann. Sci. École Norm. Sup. 28 (1911), 33-62.

8. S. Saks, Theory of the integral, 2nd ed., Monogr. Mat., Tom 7, Warszawa and Lwow, 1937.

9. C. de la Vallé-Poussin, Intégrales de Lebesgue. Fonctions d'ensembles. Classes de Baire, Paris, 1916.

Department of Mathematics, George Mason University, Fatrfax, Virotnia 22030

Current address: System Development Corporation, 7929 Westpark Drive, McLean, Virginia 22101 